\title{
The role of Precise and Imprecise Syllogisms in the di- agnosis of reasoning deficits in mental disorders
}

\author{
A. Sobrino ${ }^{1}$, M. Pereira-Fariña ${ }^{2}$ \\ ${ }^{1}$ Faculty of Philosophy. University of Santiago de Compostela (Spain) \\ ${ }^{2}$ Centro Singular de Investigación en Tecnoloxías da Información (CiTIUS). University of Santiago de Compostela
} (Spain)

1ªlejandro.sobrino@usc.es; ${ }^{2}$ martin.pereira@usc.es

\begin{abstract}
In this paper we will inquiry about the role of precise and imprecise syllogisms for diagnosing reasoning impairments in people suffering some mental disorders. Additionally, we propose some new questions that can help to better understand the already studied deficits and, perhaps, to progress in the knowledge about that paranormal way to reason.
\end{abstract}

Keywords: logic deficits, syllogisms, imprecise syllogisms, paralogic.

\section{Introduction}

Human beings are rational agents as they follow (in a broad sense) logical principles in argumentations. Arguments are frequently used to take daily decisions and to convince our interlocutors of the goodness of our conclusions. People usually argue according to logic rules, whose implementation depends on the overall 'atmosphere' surrounding its application: hidden premises, context, suppositions, beliefs, etc. These factors make human reason a quite complex task, not only linked to the application of logic principles.

Human reasoning can be altered by disease. People suffering some mental illness make arguments, but applying other logic principles that those used by healthy people. It is said that they follow paralogic or paleologic rules. While logic is the science to infer correctly, paralogic is the set of justifications used by people with mental disorders to infer conclusions.

The first systematic study of logic was due by Aristotle in Ancient Greek. He distinguished between two inference patterns: induction and deduction. Induction was generally defined as 'argument from the particular to the universal' [1] while deduction was more precisely defined as follows: A deduction is speech (logos) in which certain things having been supposed, something different from those supposed, results of necessity because of their being so.

Syllogistics [1] is the way approached by Aristotle to study deduction. A syllogism is an argument composed by two premises and a conclusion. Premises include two general terms linked by a middle one, leading to a true conclusion if the premises are also true.
In ordinary argumentation, deduction is infrequent as universal propositions or general terms are rare. However, sentences involving imprecise information are quite frequent. In this case, induction or analogical reasoning [2] plays a major rule, allowing us to infer plausible conclusions from incomplete premises. This type of reasoning can be generically labelled as approximate reasoning.

Imprecise syllogisms deal with approximate reasoning, involving arguments with incomplete premises, usually qualified by words as 'most', 'generally', etc. (e.g.; Most Spaniards are brown; almost all brown people have dark hair; therefore, most Spaniards have dark hair). They permit to achieve conclusions that are plausible, although not totally safe.

The use of syllogistic forms, both in categorical and approximate arguments, has a long tradition in the inquiry about mental disorders [8]. It is conjectured that people suffering mental pathologies show deficits in reasoning. Syllogisms are frequently used in experiments to contrast this conjecture and it is supported by good reasons: i) syllogistic moods use natural language sentences [4]; ii) syllogisms combines logical rules or principles (such as the Logic Square of Opposition [1]) and quantities (syllogistic propositions are quantified statements) in a single argument; iii) syllogisms deal with fuzzy quantities (introducing fuzzy quantifiers [5, 6], preserving its probative character; and, iv) syllogisms can be transformed into a plausible argument [6] or even a probabilistic one [7].

In this paper, we analyze the use of precise and imprecise syllogisms in the evaluation of reasoning patterns in people with mental disorders. From this analysis we propose some questions that perhaps can contribute to better classify and test reasoning impairments in schizophrenic, obsessive-compulsive or dichotomous thinkers, the most frequent types of psychiatric patients having reasoning troubles.

Thus, our aim is to propose new precise and imprecise syllogistic moods (inference patterns) as a way to deeply analyze the logical course of action of some psychiatric patients. In so far as the studies on reasoning deficits in patients with mental disorders show controversial conclusions [3], we will debate about new syllogistic forms for illuminating those disputes. Other contribution is to propose the use of classic paradoxes 
for testing the reaction of patients to an argument that combines the stiffness of the logic rules with the vagueness of the language used in the premises, such us the Sorites paradox.

In order to reach those objectives, this paper is organized as follows: in section 2, we shall describe the main models of categorical and plausible syllogisms; in section 3, several analysis about the difficulty for solving syllogistic modes are presented; in section 4, syllogistic experiments for checking the impairments of reasoning from people with mental disorders are approached; in section 5, some experiments using Soritestype arguments and fuzzy syllogisms are advanced; and, finally, in section 6, we summarize our main conclusions and some questions whose answer should contribute to illuminate this debate.

\section{Modalities of syllogism}

Syllogistic reasoning was the hegemonic logical system in West Thought from Ancient Greek to 19th century [1]. During $20^{\text {th }}$ century, it was replaced by mathematical logic, but since for quite some years it was returned to have a main role due to: i) increasing the expressiveness power by the introduction of new quantifiers in addition to all, no, some and some... not; ii) softening the probative character aiming new inference patterns for reaching conclusions in an approximate way.

\subsection{Categorical syllogism}

The main characteristic of categorical syllogisms is that they are composed by categorical statements (e.g., "All human beings are mortal”), i.e., statements that make 'assertions about classes', affirming or denying that a class (human beings) is included, totally or partially, in other (mortal). Categorical statements have three logical terms: subject-term (human-being), predicate-term (mortal) and copulative verb (are). The classic properties of categorical statements are [9]:

- Quantity: Statements can be universal, headed by all or by no, or particular, headed by some or some... not.

- Quality: Statements can be affirmative, headed by all and some, or negative, headed by no and some... not.

- Distribution: A statement distributes a term 'if it refers to each member of the class designated by that term' [1].

The combination of these properties generates the four standard categorical statements, which can be logically related in the Logic Square of Opposition (LSO) (see Figure 1) according to the following relationships of opposition:

- Contradictory (A-O, E-I): Two propositions are contradictory if they cannot both be true and they cannot both be false.

- Contrariety (A-E): Two propositions are contrary if they cannot both be simultaneously true.

- Subcontrariety (I-O): Two propositions are subcontrary if they cannot both be false.
- Subalternation (A-I, E-O): A proposition is a subalternate of other if the truthfulness of the universal one -named superaltern- entails the truthfulness of the particular one -named subaltern.

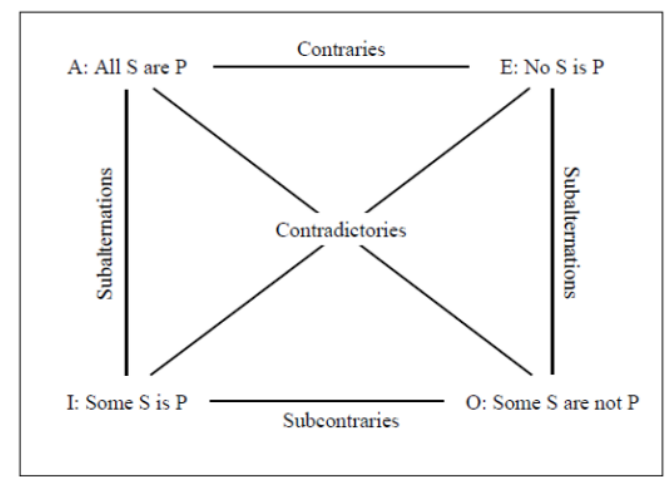

Fig. 1. Classical LSO.

Syllogism is a piece of deductive reasoning in which, using categorical statements, two general terms are linked by a middle term implying a conclusion by necessity. It is a form of mediate inference, in opposition to immediate inferences, which only include two terms and a single premise (for instance, the relationships of opposition of the LSO).

The different possible combinations among the four quantifiers (all, no, some and some... not) and the position of the middle term in the premises (see Table 1) leads to 256 different possible syllogistic inference schemes. Of them, only 24 are valid, the so-called Moods (see Table 2).

\begin{tabular}{l|l|l|l} 
Figure I & Figure II & Figure III & Figure IV \\
\hline
\end{tabular}

\begin{tabular}{l|l|l|l}
$\mathrm{Q}_{1}$ DT are MT & $\mathrm{Q}_{1}$ MT are DT & $\mathrm{Q}_{1}$ DT are MT & $\mathrm{Q}_{1}$ MT are DT
\end{tabular}

\begin{tabular}{l|l|l|l}
$\mathrm{Q}_{2}$ NT are DT & $\mathrm{Q}_{2}$ NT are DT & $\mathrm{Q}_{2}$ DT are NT & $\mathrm{Q}_{2} \mathrm{DT}$ are NT \\
\hline
\end{tabular}

\begin{tabular}{l|l|l|l}
$\mathrm{Q}_{3} \mathrm{NT}$ are MT & $\mathrm{Q}_{3} \mathrm{NT}$ are MT & $\mathrm{Q}_{3} \mathrm{NT}$ are MT & $\mathrm{Q}_{3} \mathrm{NT}$ are MT
\end{tabular}

Table 1: Figures of Aristotelian Syllogistics.

\begin{tabular}{c|c|c|c} 
Figure I & Figure II & Figure III & Figure IV \\
\hline bArbArA & cEsArE & dArAptI & brAmAntIp \\
cElArEnt & cAmEstrEs & dIsAmIs & cAmEnEs \\
dArII & fEstInO & dAtIsI & dImArIs \\
fErIO & bArOcO & fElAptOn & fEsApO \\
bArbArI & cEsArO & bOcArdO & fErIsOn \\
cElArOnt & cAmEstrOp & fErIsOn & cAmEnOs
\end{tabular}

Table 2: Moods of Aristotelian Syllogistics.

Table 3 illustrates one of the most famous Aristotelian syllogisms (in Barbara mood).

All human beings are mortal

All Greeks are human beings

All Greeks are mortal

Table 3: Example of Aristotelian Syllogism.

As previously quoted, in $20^{\text {th }}$ century Aristotelian Syllogistics was rehabilitated and new quantifiers was introduced in order to deal with added quantities. Thus, Intermediate Syllogistics [10] added 5 new quantifiers 
to the LSO (almost-all, few, most, most... not, many and many... not) and the number of valid syllogistic moods was increased from 24 to 81 . It is worth noting that these new quantifiers implied additional assumptions in the model: for instance, some, many and most means at least or no less than the quantity named (some students are blond means at least one student is blond).

Despite of this novelty in syllogistic reasoning, these additional assumptions still are crisply defined. But when the conclusion needs some degrees of tolerance, fuzzy logic has a role. Fuzzy logic approached new models for syllogistic reasoning, introducing both new forms for dealing with vague quantifiers and inference patterns. These new reasoning schemes not only include deductive patterns, but also approximate ones; i.e., arguments with more than an unique conclusion or arguments with a plausible conclusion rather than a single safe one. For instance, Zadeh's approach [5] proposes that quantifiers can be modelled as fuzzy numbers; therefore, the reasoning process is transformed into a fuzzy arithmetic calculus. Table 4 shows the basic Zadeh's example, being the quantifier of the conclusion (Most $\otimes$ Most) obtained as a fuzzy product of the quantifiers of the premises. Note that in this case, the chaining between the terms involved in the argument (students, blond and tall) is not performed by a middle term but by the logic connective and (students are blond of the first premise is the subject of the second one).

Most students are blond

Most blond students are tall

Most $\otimes$ Most students are blond and tall

Table 4: Zadeh’s Fuzzy Syllogism.

\subsection{Approximate Chaining Syllogism}

An alternative approach to approximate syllogism is to consider a non-exact chaining based on linguistic relations, obtaining a qualified conclusion.

Approximate chaining syllogism [6] preserves the standard structure of the categorical syllogism, but the role of middle term can be played by different words with similar meaning (see the example of Table 5).

All earthquakes are a disaster

No calamity is desirable

No earthquake is desirable

Table 5: Example of Approximate Chaining Syllogism.

In classical syllogistics, this type of argument would be diagnosed as a quaternio terminorum fallacy, as the link between the extreme terms (those that appear in the conclusion) is not guaranteed by and identical term. Other times, although the middle term will be played by a single term, if it is polysemic, the argument can be also incorrect. For instance, in the example of Table 5 the middle term is played by two different terms; i.e., disaster and calamity.

Nevertheless, in natural language there are a lot of words having similar meanings; i.e., synonymous. Two words are synonymous if they have equal or similar meaning; i. e., if they denote the same object in a similar way. Similarity in the meaning of the middle terms does not lead to a safe conclusion, but it should not preclude any kind of conclusion. Synonymy does not lead to valid arguments (only if the terms involved are equal, a strong case of synonymy) but to persuasive ones, based on the similarity of the terms, and offering to the speakers some degree of confidence in the conclusion. For instance, in the example of Table 5, the conclusion will be plausible for most people since disaster and $c a-$ lamity are frequently used as synonyms.

The reliability of an argument supported on synonymy or other similarity relations are expressed calculating a degree of confidence for the conclusion accordingly with the following moral: the greater the similarity between the terms, the higher the degree of confidence in the conclusion.

Similarity relations are estimated from two sources: (a) a linguistic source provides synonymy relations in the common language, (b) a mathematical source provides tools for calculating the similarity between sets of synonyms provided by the (a) source. WordNet, an electronic thesaurus, supplies the largest collection of synsets (sets of synonymous of a given word). Similarity measures (the inverse of the distance measures) offers different methods to calculate how similar are two synsets. The resemblance calculus can be adjusted or improved using other linguistic relations between terms, as antonymy, hyponymy or hyperonymy.

In Table 6, we show an example of approximate syllogism but with the conclusion qualified according to different similarity measures between the middle term ${ }^{1}$.

All fruits are vegetables

Some artificial products are food

Some artificial products are vegetables

$\left[\gamma_{\text {WordNet }}=0\right] ;\left[\gamma_{\text {Human }}=7.52\right] ;\left[\gamma_{\text {PathLenght }}=0.1\right]$

Table 6. Approximate chaining syllogism with a degree of confidence $\gamma$ on the conclusion.

\section{Levels of difficulty for solving syllogisms}

Meanwhile the object of logic are the laws of the right though, the object of the psychology of reasoning is how people effectively think, considering both right and fallacious inferences [11].

There are several studies [19] that address how people reason syllogistically. For instance, let us consider the following syllogism:

\footnotetext{
${ }^{1}$ The results for $\gamma_{\text {Human }}$ are from G.A. Miller and W.G. Charles, "Contextual correlates of semantic similarity", Language and Cognitive Processes, Vol. 6, No. 1, 1991, pp. 1-28 and for $\gamma_{\text {PathLenght }}$ are from WordNet::Similarity, http://maraca.d.umn.edu/cgibin/similarity/similarity.cgi
} 
All roses are flowers

Some flowers fade quickly

Some roses fade quickly

Table 7: Example of syllogism that most people fail.

This is a fallacious syllogism, since the conclusion does not follow from the premises, but most people classify it as valid one [11].

In [7], to test the validity of Probability heuristics model, both Aristotelian syllogisms and intermediate ones (using the quantifiers all, most, few and some... not) were analyzed. Although the study was focused on the evaluation of the model capability for predicting the results of the participants, we will only focus the hit rating classification. The achieved results indicate that, although all the arguments have the same form (two premises and a conclusion), the hit rate is very different between them. Table 8 shows the results of the analysis described in [7].

\begin{tabular}{|c|c|c|c|c|c|c|c|}
\hline \multicolumn{2}{|c|}{ Figure I } & \multicolumn{2}{c|}{ Figure II } & \multicolumn{2}{c|}{ Figure IIII } & \multicolumn{2}{c|}{ Figure IV } \\
\hline AAA & 89.87 & EAE & 88.61 & AAI & 29.11 & AAI & 16.96 \\
\hline EAE & 86.71 & AEE & 87.97 & IAI & 84.81 & AEE & 86.71 \\
\hline AII & 92.41 & EIO & 51.90 & AII & 88.61 & IAI & 91.14 \\
\hline EIO & 66.46 & AOO & 67.09 & EAO & 21.52 & EAO & 8.23 \\
\hline AAI & 5.06 & EAO & 3.16 & OAO & 68.99 & EIO & 26.58 \\
\hline EAO & 2.53 & AEO & 1.27 & EIO & 48.10 & AEO & 1.90 \\
\hline
\end{tabular}

Table 8: Meta-analysis showning the percentage of right questions by figures.

As can be observed in Table 8, Figure I shows the best results, since the moods AAI and EAO are specific case of AAA and EAE (subalternation property). Therefore, we can conclude that in this inference pattern, the right results are higher than 85\%, except in EIO mood, with $66 \%$.

In Figure II and Figure III the results are worse than in Figure I. It is worth noting that in both figures the chaining is not $\mathrm{NT} \rightarrow \mathrm{DT} \rightarrow \mathrm{MT}$, but $\mathrm{NT} \rightarrow \mathrm{DT} \leftarrow \mathrm{MT}$ in Figure II and NT $\leftarrow \mathrm{DT} \rightarrow \mathrm{MT}$ in Figure III; therefore, the property of the distribution of the quantifiers is particularly relevant and makes the inference process more complex than Figure I does. Thus, EIO of Figure II has a hit rate around $50 \%$ and none of the other moods is higher than $90 \%$. In Figure III the results are worse, since most of the moods are lower than $70 \%$.

Figure IV is the converse of Figure I; i.e., $\mathrm{NT} \leftarrow \mathrm{DT} \leftarrow \mathrm{MT}$ and this can induce some confusion from Figure I. Apart of AEE and IAI moods, the hit rates are very low, lower than $27 \%$.

Therefore, we can see as the management by human beings of syllogistic inferences shows clear differences between Figures. While Figure I has a very high percentage of correction; the remaining ones do not.

\section{Syllogistic reasoning deficits in mental disorders}

If healthy people show difficulties solving syllogisms, people with mental deficits, also do. The use of syllogistic moods has a long tradition in the inquiry about reasoning deficits in mental disorders. It is conjectured that abnormal people use paralogic principles, not logical ones, although it is controversial if paralogic rationality is a kind of deficient logic rationality or has its own principles. Syllogistic moods are used extensively to explore the logic of some mental patients.

A rational argument is made of premises and a conclusion, following the conclusion from the premises. If the conclusion does not follow from premises, the argument is incorrect or illogical. The incorrectness may be caused by two different factors: (a) the inability to determine the truth of the premises or (b) the incapability to apply logical rules. Mujica-Parodi el al. [12], suggested to follow the aforementioned distinction in order to classify the logical deficits of people with mental disorders. From now on, we will refer to them as type (a) or type (b) deficiencies.

The pioneering work addressing (b) deficits was the von Domarus' one [14]. Von Domarus aims to inquiry about 'the specific laws of logic in schizophrenia', showing that patients suffering this psychiatric illness follow some principles different to the rational ones. The key point is the logical principle of identity. In logic, the law of identity states that "each thing is the same with itself and different from another". Each object is made of a unique set of characteristic qualities or features, called by the Greeks as 'its essence'. Therefore, things that have the same essence are the same thing, and things that have different essences are different things. Accordingly von Domarus, schizophrenic patients mistake essential and accidental properties, causing fallacy inferences which conclude that two objects are identical if they share a property (and not necessarily an essential one). F. ex., from the premises: All bananas are yellow, All lemons are yellow they conclude that All bananas are lemons. Using a curious example, von Domarus reported a patient from the Insane Asylum of the University of Boston identifying Jesus and a cigarette box because both have a kind of ring surrounding them: Jesus has a circular crown around his head and the cigarette box has the precinct tax embracing its borders. Schizophrenics mistake identity based on the individual identity with similarity based on identical predicates.

Von Domarus work does not really use any syllogistic form to identify the reasoning fault or the argument quoted before. But he used a classic way to illustrate the syllogistic entailment: Venn's diagrams. He argued that, if we are rational agents, we cannot accept the conclusion that All bananas are lemons because from the fact that 'All B are $\mathrm{Y}$ ' and 'All L are $\mathrm{Y}$ ', does not involve necessarily that 'All $B$ are $L$ ', because while $\mathrm{B}$ is contained in $\mathrm{Y}$ and $\mathrm{L}$ is contained in $\mathrm{Y}, \mathrm{B}$ is not contained in L'. While a logic agent understands the anomaly of this syllogistic figure, a paralogical agent does not, accepting a defective reasoning. He concluded that this schizophrenic mode of reasoning is not a new genuine type of reasoning, but a deviation from one already well characterized.

Despite the impact of his work on further investigations, von Domarus paper was based on a serious methodological deficiency. As Arieti [15] first posed, von Domarus did not use control groups against which to 
test their claims. Then, no comparison is made between healthy and ill people and so, his conclusions lack of objectivity.

Arieti extended the investigations initiated by von Domarus and corroborated his thesis: the confusion between identity and similarity is the source of the schizophrenic reasoning deficits. But Arieti's approach introduced a substantial novelty in the syllogistic tests to which schizophrenics are faced: syllogistic forms are received differently by the psychiatric patients if they contain schematic or natural language sentences and, of these latter, if they have emotional or affective content or not. Following the Arieti's vein, Ho [16] proposed the Deductive Inference test (DIT) for diagnosing reasoning deficits. The test includes the following syllogistics types, illustrated by the corresponding examples:

A) Formal Syllogisms modes

A.1.Correct

All $A$ is $B$, all $B$ is $C$, therefore all $A$ is $C$

A.2. Fallacious

All $A$ is $B$, all $C$ is $B$, therefore, all $A$ is $C$

B) Content Syllogisms modes

B.1. Neutral

\section{B.1.1. Correct}

All sardines are fish, all fish have gills. Therefore all sardines have gills.

\section{B.1.2. Fallacious}

All sardines are fish. All tunas are fish. Therefore all sardines are tunas.

\section{B.2. Emotional}

\section{B.2.1. Correct}

All people who cause problems must be eliminated. Schizophrenic patients (like you) cause problems. Then the schizophrenic patients should be eliminated.

\section{B.2.2. Fallacious}

All schizophrenics like sports. All teachers like music. Some schizophrenics play violin.

Ho tested that schizophrenic people solve syllogisms A and B.1.1 with similar skills that healthy people (this thesis, nevertheless, is controversial), and achieves significantly better scores diagnosing the incorrectness of syllogisms type B.1.2 than the control group. However, they show poorer scores with syllogism type B.2.1, a valid argument entailing a conclusion that, changing the focus, is inadmissible from an emotional view. Finally, schizophrenics show better scores than healthy people diagnosis B.2.2 syllogisms. Goel and Bartolo [17] use syllogism with emotional content for verifying similar thesis to Arieti's one: the diagnosis about the correctness of a conclusion is highly influenced in the schizophrenic patients by the beliefs that they previously have on it. Owen et al. [18] concludes that schizophrenics show impairments concerning common-sense reasoning, prioritizing theoretical over practical rationality. If schizophrenics are faced with common-sense arguments disputing the rules of logic, they violate the rules in a lesser extent that healthy people.

Schizophrenic people also show impairments in reasoning under Mujica-Parodi (a) errors [12], i.e., those related with the difficulties to verify the truth of the premises. Schizophrenia was generally linked to delusional behavior and distortion of reality. Philips et al. [13] approached if sensory distortion influences delusional reasoning. They found that the introduction of an additional premise in an argument was disruptive both in the patient and in the control group, but distorting the first more. Kemp et al. [8] checked the difficulties of schizophrenics using heuristic rules, a common strategy from normal people to complete lacks of information in premises.

Studies about reasoning deficits in schizophrenia are not uniform with respect to their conclusions. Frequently they start from different presuppositions, but even starting from a similar view, different results emerge. Two recent papers show paradigmatically that. The Mirian et al. [3] investigation about the reasoning abilities in schizophrenia patients concludes that there is no relation between delusional symptoms and deductive impairments: logic deficits are mainly due to cognitive dysfunctions than to specific anomalies in the logic brain machinery. Their position is that depression, frequently associated with schizophrenia, provokes a lack of motivation that influences the abnormal resolution of logical tasks. Kruck et al. [20], in return, noted findings consistent with those obtained by the previous research, corroborating that patients with schizophrenia disorders have greater deficits that healthy-control patients regarding logic reasoning.

Those partially contradictory conclusions show that the 'logic of schizophrenia' is far for being complete, requiring experimentation and theory added. F. ex., if schizophrenic people choose logic before common sense, how they evaluate a commonsense reasoning? If schizophrenic people mistake similarity and identity, do they improve the reasoning ratios using similar terms instead of identical ones? Common sense or vague syllogisms could offer a still unexplored test to verify or to complete this question.

As we have seen in the previously paragraphs, deficits of deductive or categorical reasoning are strongly linked to schizophrenic people, although people with delusions show also impairments completing the information of the premises. Deficits of information in premises are strongly related to other mental illness: the obsessive-compulsive disorder (OCD). Test for performing these deficits use, as in the schizophrenia case, syllogisms, but of inductive or probabilistic types.

Many papers suggested that people suffering OCD show difficulties in reasoning about uncertain or probabilistic information [23]. Reasoning with uncertain premises, OCD people exhibit ineradicable doubts postponing a final conclusion, which at last is achieved in a suddenly and fallacious style usually called 'jumping to conclusions'. Jumping to conclusions or hasty generalization is a type of informal fallacy that consists in reaching a general conclusion from an insufficient evidence. OCD patients show a serious shortage concluding inductively, however, they do not seem to have deficiencies using deductive rules, although this thesis is controversial [23].

Inductive rationality supports risky-arguments. An argument is risky if the conclusion has any chance to be 
false even been the premises true. F. ex., I have a bag with 5 balls and extract 4 . All the pulled balls are red. Then, I infer that the following ball I will extract will be red ... but this conclusion is not sure. It is only likely. Probability is the tool used by inductive logic for evaluating risky-arguments. To infer adequately from an inductive view means to conclude proportionally to the information provided by premises. In the previous example, the probability getting a new red ball is high: $4 / 5$. But if instead four balls I had extracted only two red balls, the next to be pulled will be red with a lower probability: $2 / 5$.

Linney et al. [24] investigated about the reasoning deficit of delusional patients concerning testing hypothesis and probability judgments. Using different tests, they conclude that a big amount of information is required by delusional patients before validate a hypothesis. They also checked a deficiency making metaprobabilities sentences, as f. ex., It is very likely that an investor bets to an unlikely value at some point.

In the inquiry about the possible deficits of OCD patients regarding inductive reasoning, syllogisms have a leading role, as the paper by Simpson et al. [22] shows. Simpson et al. noted that significant differences exist in the way the OCD patients reason with syllogisms: While OCD and control people show similar abilities with neutral deductive arguments, people with OCD show differences reasoning with syllogisms including emotional (believable/unbelievable) content.

A main characteristic of OCD patients is a pathological perfectionism. Patients with dysfunctional perfectionism reach a high score on the Intolerance of Uncertainty Questionnaire (IUQ), as O’Connor et al. [23] recently pointed out. People that show high IU require a greater number of cases before performing a decision, but if they are unable to collect all the necessary cases, a lower threshold assessing ambiguity or vagueness precipitate a decision in a jumping to conclusion style.

No A are B

Some C are B

Therefore, some $\mathrm{C}$ are not $\mathrm{C}$

Table 9. Example of Syllogism used by Simpson et al. [22].

OCD people were faced to inductive syllogisms in which a conclusion should be inferred from the information provided by premises. But inductive syllogisms are only a (small) part of imprecise ones. As previously quoted, probabilistic, fuzzy or linguistically chained are other well-known modalities of approximate syllogisms. Their inclusion in the experiments to test the impairment of OCD people reasoning can help to get new knowledge about the subject. Particularly, there are probabilistic and fuzzy syllogisms that permit to conclude in a safe manner, even within and interval or range of possibilities. To test if OCD patients show (or not) in those cases some kind of pathological indecision is an attractive task. On the other hand, to check the degree of secureness of OCD people concluding in an inevitable vague manner in a approximate chained syllogism could help to verify the strength of their disorder.
Finally, a brief note about other psychiatric disorder: dichotomous thinking. As Oshio [21] highlighted, dichotomous people have the following characteristics: (a) preference for dichotomy: patients direct their thought towards distinctness and clarity; not to ambiguity and obscuration; (b) dichotomous belief: all things of the world are divided in a extremist or Manichaean stile: black/white; good/bad; true/false; (c) profit-andloss thinking: they urge to gain access to benefits and to avoid disadvantages, although Oshio proved that this category admits certain degree of flexibility, and so it is not a clear feature of that personality disorder.

Although dichotomous thinking should show deficiencies reasoning inductively, no paper using probabilistic syllogisms has approached this question. There is not also any inquiry into the possible relationship between the dichotomous thinking and crisp reasoning. Facing dichotomous patients to crisp and approximate syllogisms, both in its necessary (within a range) or plausible modalities, should help better know how to reason these patients.

\section{The role of imprecise syllogisms characterizing impairments in mental disorders}

Reasoning, being rational or irrational, is what human beings require to substantiate or justify the conclusions of their arguments. If people do not think rationally, their behavior will be strange or alien for a rational agent. Reasoning is logic but general people do not use classic logic in their daily arguments. Plausible inferences support the main part of our daily decisionmaking activity.

Although reasoning is mainly logic, not all the reasoning process rests in logic. Content (emotive or not), presuppositions, beliefs, heuristics, background, context and a myriad of factors are involved, not in the correctness of arguments, but in their acceptance. Investigations previously quoted have shown the influence of emotional biased sentences in classical or inductive syllogistic argumentations, both in control and patient groups. Extra-logic factors greatly contribute to arguments being well accepted in the everyday reasoning. And logic is not only classic logic. Ordinary arguments are essentially vague instead of crisp, and other logics, as fuzzy logic, enter in scene.

As a main characteristic of the human mind is the management of imprecise information, imprecise syllogisms can contribute to better explain some aspects of the paralogical reasoning. Relating the problem of vagueness, two issues still not reported in the literature about the logic deficits of people with mental disorders, deserves to be addressed.

\subsection{Sorites paradox applied to dichotomous think- ing patients.}

Sorites is an ancient paradox based on the use of vague predicates, as heap or bald. A type-Sorites reasoning permits to move from a true premise, using a logical rule, to a false conclusion, something forbidden in 
logic. An illustration of this paradox in a polysyllogistic form is the following:

All accumulation of $10^{10}$ grains of sand is a heap All accumulation of $10^{10}$ minus 1 grain is indistinguishable from an accumulation of $10^{10}$ grains of sand.

Therefore, All accumulation of $10^{10}$ minus 1 grain is a heap.

All accumulation of $10^{10}-1$ grains of sand is a heap All accumulation of $10^{10}-1$ minus 1 grain is indistinguishable from an accumulation of $10^{10}-1$ grain of sand

Therefore, all accumulation of $10^{10}-1$ minus 1 grain is a heap.

....

Iterating this chain of argumentations we can conclude that

All accumulation of 0 grains of sand is a heap,

a conclusion that is obviously false.

Due to its paradoxical status, Sorites reasoning causes a mental state of uneasiness to healthy people. But, how a dichotomous reasoning patient (with respect to a control group) faces this argument? A Soritex paradox argument allows, in each step, a crisp detachment that in the end falls into a very false conclusion.

\subsection{Probabilistic (undetermined but crisp) and vague syllogisms applied to OCD patients}

Statistical syllogism is a type of inductive syllogism in which the conclusion follows from premises in a given proportion. It satisfies the following abstract pattern:

$$
\begin{aligned}
& 1 \% \text { of } F \text { are } G \\
& \text { 2. } X \text { is an } F \\
& \text { Therefore, } \% X \text { is a } G
\end{aligned}
$$

\section{F. ex., $\quad 1.20 \%$ of Portuguese are blond \\ 2. $X$ is Portuguese \\ 3. $X$ is blond with probability 0.2}

Note that, although statistical syllogisms do not conclude with certainty, they are not vague. In the previous example, the probability of finding a blond Portuguese is 0.2 ; not 0.4 or another value. Vague syllogism uses vague quantifiers. F. ex.

\section{Most of F are $G$ \\ 2. Few E are $F$ \\ 3. Conclusion?}

Depending on the cardinality of E, we can conclude that 'very few', 'few' or even 'many' E are G. In this case, the proportion of $E$ that are $G$ is not a fixed number, but a linguistic label represented by a fuzzy number.

OCD patients show the same behaviour using statistical syllogisms and vague syllogisms? OCD patients use vague quantifiers to reasonably conclude about uncertain syllogisms or jump to an all/nothing-conclusion as happen in inductive generalization?

\section{Concluding remarks}

Psychiatry studies the connection between varieties of reasoning and pathological thinking, based on the belief that people behavior is influenced by the way that they think. If the behavior is abnormal, perhaps knowing how is the logic that supports this conduct can help to apply a cognitive therapy for correcting deviant thoughts, although this thesis is controversial for cognitive therapists.

The misuse of logic is not an exclusive characteristic of people with mental disorders. But a difference exists between healthy and ill people: if an argument shows doubts about its correction, after some logician explains us the validity of logical pattern underlying it, we must admit that it is valid and the conclusion even obvious. In other case, as von Domarus said, our mind is not logic and not healthy.

Arguments in ordinary language are rarely crisp and very abundantly imprecise or vague. Classic logic traditionally deals with crisp inferences, but vague inferences are managed by the logics of uncertainty, inductive and fuzzy logic at the head.

A typical way to schematize an argument is to put it as a syllogism. There are several types of syllogisms, being the most common the categorical and inductive ones. Classic and inductive syllogisms have been used extensively in the study of reasoning deficits in people with mental disorders. But another types of syllogism can be used in that task: fuzzy syllogisms or approximate chaining syllogisms are modalities of syllogism in which people can conclude in an approximate form, either within intervals of degrees, or chaining middle terms under similarity linguistic relations as synonymy.

People suffering mental disorders that paradigmatically show reasoning deficits are schizophrenics, obsessive-compulsive disorder people or dichotomous thinkers. The following are some questions that can be explored in that collective if fuzzy syllogisms or approximate chaining syllogisms were employed in the experiments:

- The 'logic of schizophrenia' is far for being complete, requiring experimentation and theory added. If schizophrenic people choose logic before common sense, how they evaluate a commonsense reasoning? If schizophrenic people mistake similarity and identity, do they improve the reasoning ratios using similar terms instead of identical ones? Common sense or vague syllogisms could offer a still unexplored test to verify or to complete this question.

- The inclusion of fuzzy or linguistically chained syllogisms in the experiments to test the impairment of OCD people reasoning can contribute to get new knowledge about this topic. Particularly, probabilistic and fuzzy syllogisms permit to conclude in a safe manner, even within an interval or range of possibilities. To test if OCD patients show (or not) in those cases some kind of pathological indecision is an attractive task. On the other hand, to check the degree of secureness of OCD people concluding in an inevitable vague 
manner in a chained reasoning syllogism could help verify the strength of their disorder.

- Because there is not any work about the possible relationship between the dichotomous thinking and crisp reasoning, facing dichotomous thinkers to crisp and approximate syllogisms, both in its necessary (within a range) or plausible forms, should help better know how argue these patients.

Finally, to test experimentally with normal and abnormal people how they face Sorites arguments, characterized by having a supposed crisp detachment founded on premises having vague predicates, should illuminate the difference between sick and healthy people attending some point still unexplored.

\section{Acknowledgement}

This work was supported in part by the European Regional Development Fund (ERDF/FEDER) under the projects CN2012/151 of the Galician Ministry of Education and by the Spanish Ministry of Economy and Competitiveness under grant TIN2011-29827-C02-02.

\section{References}

[1] Aristotle, Prior and posterior analytics, Clarendom Press, 1949.

[2] G. Polya, Mathematics and Plausible Reasoning: Induction and Analogy in Mathematics V. 1, Princenton University Press, 1990.

[3] D. Mirian, et al. Exploring logical reasoning abilities in schizophrenia patients, Schizophrenia Research 127:178-180, 2011.

[4] F. Sommers, The Logic of Natural Language, Clarendon Press, 1982.

[5] L. A. Zadeh, Syllogistic reasoning in fuzzy logic and its applications to usuality and reasoning with dispositions, IEEE Transactions On Systems, Man and Cybernetics, 15:754-765, 1985.

[6] M. Pereira-Fariña, A. Sobrino and A. Bugarín, A Proposal of Fuzzy Chained Syllogism based on the Concept of Synonymy. Proc. 2013 International Fuzzy Conference on Fuzzy Systems (FUZZ-IEEE 2013), 1-8, 2013.

[7] M. Oaksford and N. Chater, Bayesian Rationality. The probabilistic approach to human reasoning, Oxford University Press, 2007.

[8] Kemp, R. et al. Reasoning and delusions, British J. of Psychiatry, 1997, 170, 398-405

[9] I. M. Copi, Introduction to logic, MacMillan, 1994.
[10] P. L. Peterson, Intermediate Quantities. Logic, linguistics, and Aristotelian semantics, Ashgate, 2000.

[11] D. Kahneman, Thinking, Fast and Slow, Farrar, Straus and Giroux, 2011

[12] Mujica-Parodi, L. et al. Logical Processing, Affect and Delusional Thought in Schizophrenia. Harvard Rev. Psychiatry, 8(2): 73-83, 2000.

[13] Phillips, M. L. A Cognitive neuropsychological approach to the study of delusions in late-onset schizophrenia, Int. J., of Geriatric Psychiatry, 12:892-901, 1997.

[14] E. von Domarus, The specific laws of logic in schizophrenia, in J. S. Kasanin (ed.), Language and Thought in schizophrenia. Berkeley. Univ. of California Press, 1944

[15] S. Arieti, Interpretation of schizophrenia. N.Y., Brunner, 1955.

[16] D. Y. F. Ho, Modern logic and schizophrenia thinking, Genetic Psychology Monographs, 89: 145-165, 1974.

[17] V. Goel and A. Bartolo, Logical reasoning deficits in schizophrenia. Schizophrenia Research, 8788, 2004.

[18] G. S. Owen et al., Are people with schizophrenia more logical that healthy volunteers? British $J$. of Psychiatry, 191:453-454, 2007.

[19] P. Johnson-Laird, .The Psychology of Syllogisms, Cognitive Psichology, 10, 64-99, 1978

[20] C. L. Kruck et al., Inferential-Reasoning Impairment in Schizophrenia-Spectrum Disorders, $J$. Neuropychiatry Clin. Neuroscience, 23:2, 211-214, 2001.

[21] A. Oshio, An all-or-nothing thinking turns into darkness: relations between dichotomous thinking and personality disorders, Japanese Psychological Research, 54 (4):424-429, 2012.

[22] J. Simpson et al., Reasoning in people with obsessive-compulsive disorder, British J. of Clinical Psychology, 2007, 46:397-411, 2007.

[23] K. P. O’Connor, M-C. Pélissier, Reasoning in Anxiety, OCD and Related Disorders: Can formal Reasoning inform us about psychopathology?, In Selek, S. (ed.), Different views of anxiety disorders. InTech, 2011.

[24] Y. M. Linney et al., Reasoning biases in delusion-prone individuals, British J. of Clinical Psychology, 37:285-302, 1998. 